OPEN ACCESS

Edited by:

Zhigang Liu,

Northwest A\&F University, China

Reviewed by:

Yashi Mi,

University of Arizona, United States

Guoyuan Qi.

University of Arizona, United States

${ }^{*}$ Correspondence:

Chao Ma

machao@ibms.cams.cn

Xiuhua Zhang

zhangxh@pumch.cn

tThese authors have contributed equally to this work

Received: 17 January 2021 Accepted: 07 April 2021 Published: 28 May 2021

Citation:

Cui H, Su W, Cao Y, Ma L, Xu G,

Mou W, Zhang $H, Y u J, M a C$, Zhang $X$ and Huang $Y$ (2021) Lack of Spinal Neuropeptide Y Is Involved

in Mechanical Itch in Aged Mice.

Front. Aging Neurosci. 13:654761.

doi: 10.3389/fnagi.2021.654761

\section{Lack of Spinal Neuropeptide Y Is Involved in Mechanical Itch in Aged Mice}

\author{
Huan Cuiit, Wenliang Su${ }^{2 t}$, Yan Cao ${ }^{1 t}$, Lulu $\mathrm{Ma}^{2}$, Guangyan $\mathrm{Xu}^{2}$, Wanying $\mathrm{Mou}^{2}$, \\ Hanlin Zhang ${ }^{1}$, Jiawen $\mathrm{Yu}^{2}$, Chao Ma ${ }^{1,3 *}$, Xiuhua Zhang ${ }^{2 *}$ and Yuguang Huang ${ }^{2}$ \\ ' Department of Human Anatomy, Histology and Embryology, Neuroscience Center, Institute of Basic Medical Sciences, \\ School of Basic Medicine, Chinese Academy of Medical Sciences, Peking Union Medical College, Beijing, China, \\ ${ }^{2}$ Department of Anesthesiology, Peking Union Medical College Hospital, Chinese Academy of Medical Sciences, Peking \\ Union Medical College, Beijing, China, ${ }^{3}$ Chinese Institute for Brain Research, Beijing, China
}

Neuropeptide $Y$ (NPY) signaling plays an essential role in gating the pruritic afferent information in the spinal cord. Recent studies revealed that the aging process downregulated the expression of NPY in the central nervous system. We propose that the lack of spinal NPY may be involved in certain types of pruritus in the elderly population. This study was designed to investigate the role of NPY in aging-induced itch using the senile mouse model. The expression of NPY in the spinal dorsal horn was compared between young ( 2 months old) and aged (24 months old) mice. Western blotting and immunohistochemistry showed that the expression of NPY was significantly reduced in the spinal dorsal horn in aged mice. In addition, a neuronal maker of apoptosis, TUNEL, was detected in the NPY positive neurons only in the aged spinal cord. Behavioral assay indicated that light mechanical stimulus evoked significantly more scratching in the aged than in the young mice, whereas chemical-evoked itch and painrelated behaviors were not altered. Intrathecal injection of either NPY or LP-NPY, a NPY receptor 1 (NPY1R) agonist, significantly alleviated the mechanically evoked itch in aged mice without altering the responses to chemical pruritogens. Our study suggested that downregulation of spinal NPY in the aged mice might play a role in the higher incidence of the mechanically evoked itch than that in the young mice. Therapies targeting the NPY system might serve as a potential strategy for alleviating the pruritic symptoms among the elderly population.

Keywords: neuropeptide Y, mechanical itch, spinal dorsal horn, senile pruritus, NPY1R

\section{INTRODUCTION}

Pruritus is an increasingly concerning clinical problem, which frequently occurs in older people and causes needless suffering of patients' lives. Senile pruritus is defined as chronic pruritus, which lasts 6 or more weeks in a person over 65 years old with elusive causes determined by appropriate examination (Ward and Bernhard, 2005; Valdes-Rodriguez et al., 2015). Previous studies showed a wide prevalence of senile pruritus, almost one-third in a nursing home in America. Increasing along with age, senile pruritus might be a part of the aging process (Chang et al., 2013; Clerc and Misery, 2017).

Abbreviations: NPY, neuropeptide Y; NPY1R, neuropeptide Y receptor 1; TLR5, Toll-like receptor 5; Ucn3: Urocortin 3. 
There are mainly two forms of itch: chemical itch irritated by chemical materials, such as histamine and chloroquine, and mechanical itch evoked by light mechanical stimuli (Fukuoka et al., 2013; Bautista et al., 2014; Koch et al., 2018). In addition, another type of itch can be evoked by audiovisual itch stimuli, which is assigned to contagious itch (Yu et al., 2017). The pathologic pruritus induced by innocuous mechanical stimuli is defined as "alloknesis" (Akiyama et al., 2012). Senile pruritus is usually associated with the dry skin condition and is always evoked by light mechanical stimulation, therefore presenting more likely as alloknesis (Berger et al., 2013; Clerc and Misery, 2017). The mechanism of senile pruritus remains unknown. Skin aging, charactered by the drier skin and weaker barrier, was mainly concerned in previous studies. Besides, the age-related loss of mechanosensitive Piezo2 channel in Merkel cells also produced alloknesis, suggesting that alteration in sensory element was involved in the senile pruritus (Feng et al., 2018).

Neuropeptide Y (NPY), a linear polypeptide with 36 amino acid residues, is one of the most abundant peptides in the central nervous system (CNS) (Tatemoto et al., 1982). The NPY system's dysfunction is related to the cellular hallmarks of aging, including loss of proteostasis, deregulated nutrient sensing, mitochondrial dysfunction, cellular senescence, stem cell exhaustion, and altered intercellular communication (Tatemoto et al., 1982; Silva et al., 2005; Hsieh et al., 2013). Recent studies also indicated the specific role of spinal NPY in gating mechanical itch signal, but not chemical itch signal. The NPY ${ }^{+}$ interneurons formed an inhibitory pathway from hairy skin to suppress mechanical itch (Bourane et al., 2015). Furthermore, spinal $\mathrm{NPY}^{+}$interneurons gated mechanical itch by inhibiting Urocortin $3^{+}\left(\mathrm{Ucn}^{+}\right)$excitatory interneurons that received peripheral inputs from Toll-like receptor $5^{+}\left(\mathrm{TLR}^{+}\right) \mathrm{A} \beta$ lowthreshold mechanoreceptors (LTMRs) (Pan et al., 2019). In addition, NPY receptor 1 (NPY1R) signaling within the dorsal horn suppressed the activity of excitatory interneurons and gated mechanical itch after challenged by NPY (Acton et al., 2019).

However, the involvement of spinal NPY in senile pruritus is still unclear. Here, we sought to address the role of spinal NPY in aging-related alloknesis and alleviate senile pruritus through modulating the spinal NPY system. We hypothesized that aging induced the deficiency of NPY in the spinal dorsal horn, thereby evoking alloknesis in aged mice. We tested our hypothesis by observing alloknesis-related behavior in aged mice together with pharmacological intervention to NPY and NPY1R. Our findings revealed a neural mechanism for alloknesis in aged mice, suggesting potential therapeutic strategies for senile pruritus.

\section{MATERIALS AND METHODS}

\section{Animals}

C57BL/6 mice (2 months old and 2 years old, 20-30 g, provided by HFK Bioscience Co., Ltd, Beijing, China) were housed in a controlled environment $\left(21 \pm 4^{\circ} \mathrm{C}\right.$, standard 12 -h light/dark cycle, $4-5$ mice per cage). All animal experiments were approved by the Institutional Animal Care and Use Committee in the Chinese Academy of Medical Sciences, Institute of Basic
Medical Sciences (approval number: \#211-2014). Both male and female mice were used in all studies. Animals were randomized to experimental groups, and no sex differences were noted. The gender of used mice in each group was included in the figure legends.

\section{Drug Administration}

To observe the effect of neutralizing spinal NPY, rabbit isotype control IgG ( $5 \mu \mathrm{g}$ in $5 \mu \mathrm{l}$ sterile saline; Cell Signaling Technology) and rabbit anti-NPY IgG $(5 \mu \mathrm{g}$ in $5 \mu$ l sterile saline; Cell Signaling Technology) were intrathecally administrated $1 \mathrm{~h}$ prior to each behavioral test. NPY (Tocris) and [Leu31, Pro34]-NPY (LP-NPY, a selective agonist for NPY1R; Tocris) were dissolved in $0.9 \%$ sterile saline (vehicle) (Fuhlendorff et al., 1990). Both NPY $(0.5 \mu \mathrm{g}$ in $5 \mu \mathrm{l}$ sterile saline) and LP-NPY $(10 \mu \mathrm{g}$ in $5 \mu \mathrm{l}$ sterile saline) were intrathecally injected $15 \mathrm{~min}$ before each behavioral test (Hua et al., 1991; Acton et al., 2019). Both histamine and chloroquine were dissolved in sterile saline. Each drug injected was previously prepared and then coded by a laboratory assistant and not the experimenter. One experimenter who operated the drug injection was blinded to the code, and thus the chemical injected, as was another observer who carried out the behavioral tests.

\section{Mechanical Alloknesis Test}

Our behavioral test followed a previous study (Feng et al., 2018). Briefly, mice were fully acclimated by being placed in the test chamber $1 \mathrm{~h}$ quaque die (QD) for 3 consecutive days before each behavioral assay. The fur on the nape of the neck was shaved without any skin lesions. Von Frey filaments ranging from 0.008 to $1.0 \mathrm{~g}$ were used to deliver mechanical stimuli. The filaments touched the skin and held on for up to $1 \mathrm{~s}$ unless the mice scratched the shaved skin with their hindpaw. Five stimuli were delivered for each filament with a 10-s interval between adjacent two weights. The total response number to each filament was counted to evaluate the degree of mechanical itch.

\section{Von Frey Test}

According to previous reports, mice were placed on the wire mesh for 3 consecutive days to adapt to the environment before the test. The plantar surfaces of the hindpaw were stimulated with defined von Frey filaments ( 0.16 and $0.4 \mathrm{~g}$ ) for five times with an interval of $10 \mathrm{~min}$. The percentage of withdrawal response was calculated.

\section{Acute Itch Behavior}

The nape of the neck was shaved for the tested mouse. The mice acclimated to the same chamber for 3 consecutive days. Histamine (200 $\mu \mathrm{g}$ in $10 \mu \mathrm{l}$ normal saline per mouse) or chloroquine ( $50 \mu \mathrm{g}$ in $10 \mu \mathrm{l}$ normal saline per mouse) was applied to the shaved skin by intracutaneous injection. Immediately after each injection, mice were put into the chamber and videotaped for $30 \mathrm{~min}$. The videotapes were played back, and an observer blinded to the treatments and groups counted the number of scratching bouts directed toward the injection sites. 


\section{Immunohistochemistry and Immunofluorescence Staining}

Under deep anesthesia with isoflurane, mice were perfused through the left ventricle with sterile $0.1 \mathrm{M}$ phosphate-buffered saline (PBS), followed by pre-cooled $4 \%$ paraformaldehyde. The cervical spinal cords (C1-C3) were isolated. After a dehydrated overnight in $30 \%$ sucrose, tissues were embedded in OCT (Tissue-Tek) and cut into $15-\mu \mathrm{m}$ thickness sections. Then, sections were permeabilized with $0.3 \%$ Triton X-100 and treated with microwave heat-induced antigen retrieval $\left(95^{\circ} \mathrm{C}, 15 \mathrm{~min}\right)$. Furthermore, 5\% hydrogen peroxide was applied to block the endogenous peroxidase. Then, sections were incubated with primary antibody (rabbit anti-neuropeptide Y, 1:400, Cat: 11976; Cell Signaling Technology) at $4^{\circ} \mathrm{C}$ overnight. After rinsing with PBS, sections were incubated with the second antibody (horseradish peroxidase-labeled goat anti-rabbit IgG, PV-9001; ZSGB-Bio, Beijing, China) and colored with diaminobenzidine $(\mathrm{DAB})$ solution. Images were captured using a laser confocal microscopic imaging system (FV1000 and Olympus FluoView software; Olympus, Japan). ImageJ software was used to analyze the expression of NPY based on the color intensity. Quantitative analysis was determined by analyzing three spinal cords from young and aged mice (three sections per spinal cord).

For the immunofluorescence staining, sections were permeabilized with $0.3 \%$ Triton X-100, blocked for $1 \mathrm{~h}$ at room temperature with $10 \%$ donkey serum, and then incubated overnight at $4^{\circ} \mathrm{C}$ with primary antibody (rabbit antineuropeptide Y, 1:400, Cat: 11976; Cell Signaling Technology). After washing three times with PBS, sections were incubated by secondary antibody (Alexa Fluor 488-conjugated donkey anti-rabbit, 1:400, Cat: A-21206; Invitrogen, United States) for $1 \mathrm{~h}$. From originally described in 1992, TUNEL staining has been used for localizing apoptotic DNA fragmentation in situ and detecting cell death in vivo (Gavrieli et al., 1992). Sections were washed in a darkroom and then incubated with the mixture of terminal deoxynucleotidyl transferase and Cy3-conjugated dUTP (One Step TUNEL Apoptosis Assay Kit; Beyotime, China) for $1 \mathrm{~h}$. The slides were then washed in PBS and coverslipped with VECTASHIELD Mounting Medium with 4,6-diamidino2-phenylindole (DAPI, ZSGB-Bio, Beijing, China). The images were captured using a laser confocal microscopic imaging system (FV1000 and Olympus FluoView software; Olympus, Japan). Three non-adjacent spinal cord sections were randomly selected for each mouse, and three mice were included for each group. The TUNEL ${ }^{+}$cells within the right spinal dorsal horn were counted for each section to evaluate the apoptotic condition. In addition, the percentages of $\mathrm{TUNEL}^{+} \mathrm{NPY}^{+}$cells among $\mathrm{NPY}^{+}$ neurons within the right spinal dorsal horn were summarized to evaluate the apoptotic condition of $\mathrm{NPY}^{+}$neurons.

\section{Western Blot}

The cervical spinal cords $(\mathrm{C} 1-\mathrm{C} 3)$ in each group were excised. The samples were homogenized in T-PER Tissue Protein Extraction Reagent (Thermo Fisher Scientific, United States) containing Protein Phosphatase Inhibitor (Solarbio, Beijing, China) and phosphatase inhibitor Cocktail (CWBio, Beijing,
China). The lysates were then homogenized and centrifuged $\left(12,000 \times g\right.$ for $15 \mathrm{~min}$ at $\left.4^{\circ} \mathrm{C}\right)$. The protein samples were then separated by sodium dodecyl sulphate (SDS)-polyacrylamide gel electrophoresis and transferred to a polyvinylidene fluoride (PVDF) membrane (Thermo Fisher Scientific, United States). The membranes were blocked in $5 \%(\mathrm{w} / \mathrm{v}) \mathrm{BSA}$ for $1 \mathrm{~h}$ at room temperature and incubated with primary antibodies (rabbit antineuropeptide Y, 1:1,000, Cat: 11976; Cell Signaling Technology; mouse anti- $\beta$-actin, 1:1,000, Cat: CW0096; CWBio) overnight at $4^{\circ} \mathrm{C}$, followed by incubation with corresponding secondary antibody for $1 \mathrm{~h}$ at room temperature. The bands were scanned with Tanon 5800 Luminescent Imaging Workstation (Tanon Science \& Technology Co., Ltd. Shanghai, China) by Highsig ECL Western Blotting Substrate (Solarbio, Beijing, China). The band intensity was measured by ImageJ software (National Institutes of Health, Bethesda, MD, United States).

\section{Data Analysis}

All data were presented as the mean and its standard error (mean \pm SEM). Statistical analyses were performed using the SPSS software (version 17.0). Shapiro-Wilk test was applied to determine the normality for the parametric test. Differences between the two groups were analyzed using Student's $t$-test. A statistically significant difference was defined as a two-sided $P$ value $<0.05$.

\section{RESULTS}

\section{Aging Downregulates the Expression of NPY in the Spinal Cord}

The expression of spinal NPY was compared between young and aged mice. Western blotting revealed that the aging process significantly down-regulated NPY in the spinal dorsal horn $(P=0.0006$, Figures $1 \mathrm{~A}, \mathbf{B})$. The immunohistochemistry results showed that NPY was distributed mainly in the dorsal horn's surface layer and the myelocoele area. The content of NPY in the spinal dorsal horn was reduced in the aged mice compared with the young mice $(P<0.0001$, Figures 1C-E). The TUNEL staining in the aged mice showed more $\mathrm{TUNEL}^{+}$cells than that in the young mice $(P<0.0001$, Figures $1 \mathbf{F}-\mathbf{H})$. In addition, the immunofluorescence staining showed that part of the $\mathrm{NPY}^{+}$cells was positive for TUNEL staining. Moreover, the percentage of TUNEL $^{+}$cell among the $\mathrm{NPY}^{+}$cells was increased in the aged mice compared with the young mice $(P<0.0001$, Figure 1I).

\section{Aging Induces Alloknesis Without Affecting the Chemical Itch}

Firstly, acute itch in response to classical pruritogens was assessed in young and aged mice. The scratching responses to histamine injection were not altered in the aged mice, suggesting that aging did not affect the histamine-dependent itch $(P=0.63$, Figure 2A). Moreover, scratching response to intradermal injection of chloroquine was unaffected by aging, indicating the normal histamine-independent itch in aged mice $(P=0.51$, Figure 2B). The response to mechanical pain stimulation toward 

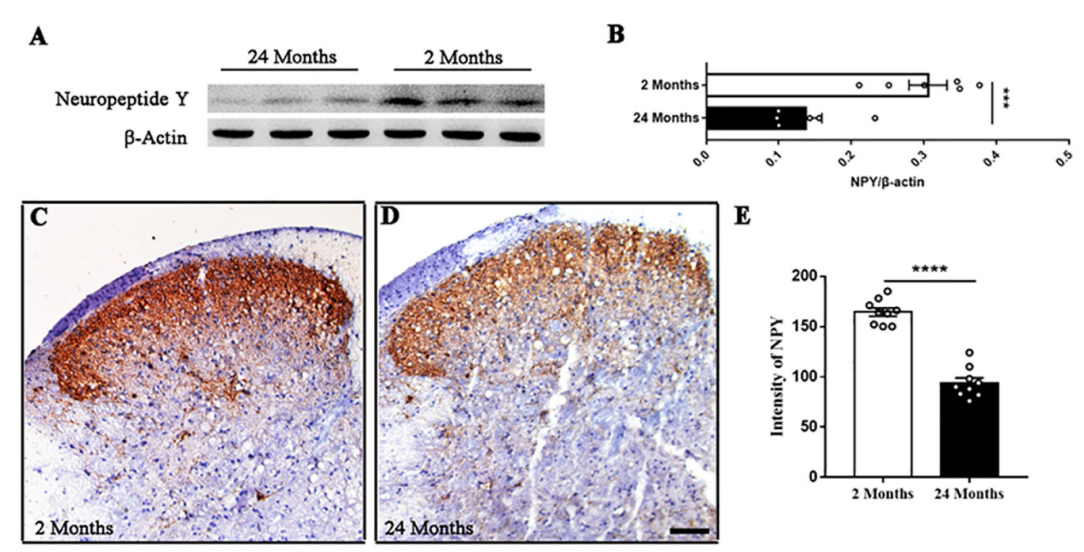

$\mathbf{E}$
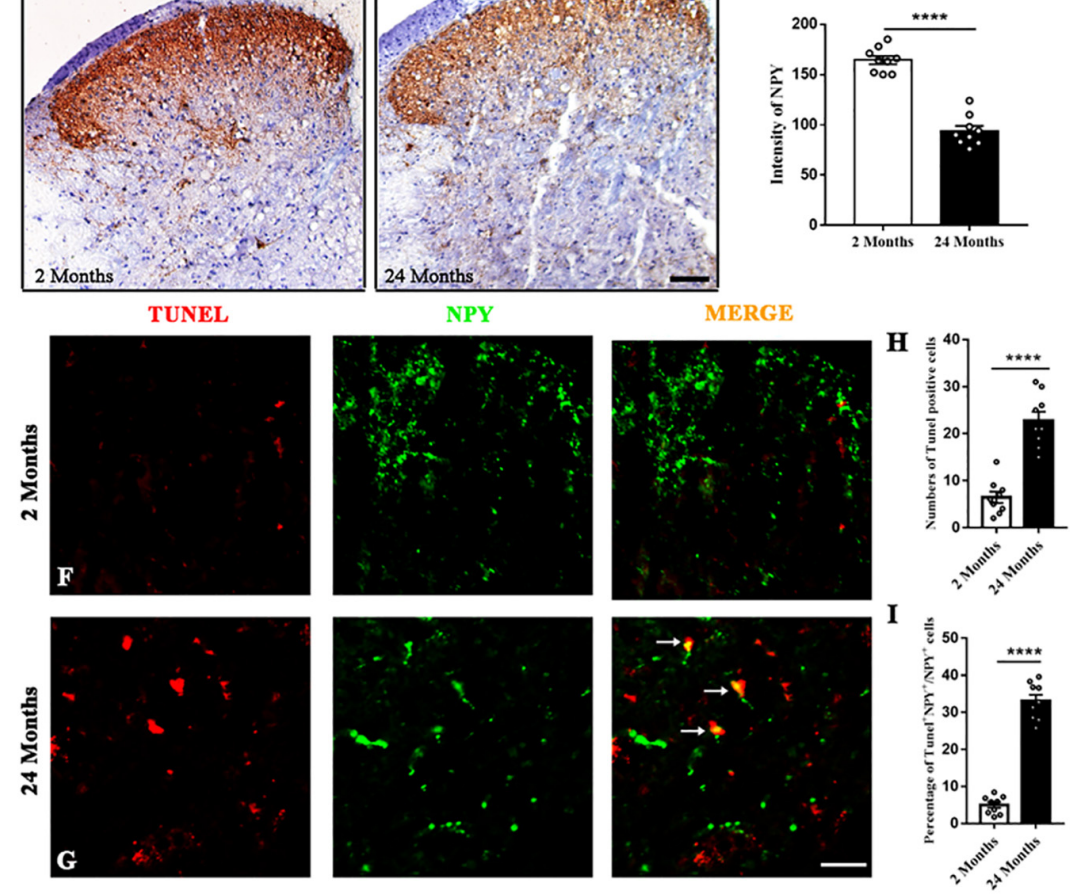

FIGURE 1 | The degeneration of spinal NPY in aged mice. (A,B) Aging down-regulated NPY in the spinal dorsal horn. Six mice in each group, ${ }^{\star \star \star \star} P<0.0001,2$ (3 male and 3 female) versus 24 months (3 male and 3 female), Student's $t$-test. (C,D) The distribution of NPY in the spinal dorsal horn of young (C) and aged (D) mice. Scale bar: $100 \mu \mathrm{m}$. (E) Quantitative analysis of NPY intensity indicated the degenerated spinal NPY in aged mice compared with the young mice. Nine slices from 3 mice in each group, ${ }^{\star \star \star} P<0.001,2$ (2 male and 1 female) versus 24 months ( 2 male and 1 female), Student's $t$-test. (F,G) Colocalized cells (yellow, arrows) by TUNEL (red) and NPY (green) fluorescence in young and aged mice. Scale bar: $50 \mu \mathrm{m}$. (H) The number of TUNEL ${ }^{+}$cells in each dorsal horn of young and aged mice. Nine slices from 3 mice in each group, ${ }^{\star \star \star \star} P<0.0001,2$ (2 male and 1 female) versus 24 months ( 2 male and 1 female), Student's $t$-test. (I) The percentages of TUNEL ${ }^{+}$cells among NPY+ cells in the spinal dorsal horn of young and aged mice. Nine slices from 3 mice in each group, ${ }^{\star \star \star \star} P<0.0001,2(2$ male and 1 female) versus 24 months (2 male and 1 female), Student's $t$-test.

the plantar surfaces of the hindpaw was also observed. Von Frey test $(0.16$ and $0.4 \mathrm{~g})$ did not reveal any difference in the aged mice relative to the young mice $(0.16 \mathrm{~g}: P=0.66,0.4 \mathrm{~g}: P>0.99$, Figures 2C,D). However, the mechanical stimulation to the nape skin by von Frey filaments, specifically the $0.04(P=0.0086), 0.07$ $(P<0.0001)$, and $0.16 \mathrm{~g}(P=0.00019)$ filaments, induced more scratching behavior in the aged mice (Figure 2E). These results indicated that aging sensitized mechanical itch in response to the light mechanical stimulation without affecting the acute chemical itch and mechanical pain.

\section{Neutralizing Spinal NPY Intensifies Alloknesis in Young and Aged Mice}

To investigate the effect of NPY deficiency, the neutralizing antibody was applied, and the relative behavioral outcomes were observed. For the young mice, the intrathecal injection of anti-NPY antibody induced alloknesis when stimulated by light mechanical stimulus $(0.04 \mathrm{~g}: P=0.023,0.07 \mathrm{~g}: P=0.028$, and $0.16 \mathrm{~g}: P=0.0044)$ compared with the isotype IgG (Figure 3A). However, the scratching response to chemical stimulus (histamine: $P=0.88$ and chloroquine: $P=0.75$ ) was not altered by the injection of anti-NPY antibody (Figures 3B,C). In addition, the behavioral effect of the anti-NPY antibody was evaluated in aged mice. Compared with the isotype IgG, the anti-NPY antibody increased the scratching behavior in aged mice receiving light mechanical stimulus (0.04 g: $P=0.0086$ and $0.07 \mathrm{~g}: P=0.023$ ) (Figure 3D). Meanwhile, the scratching behavior in response to chemical agents (histamine: $P=0.74$ and chloroquine: $P=0.68$ ) showed no difference for the aged mice receiving isotype IgG and anti-NPY antibody (Figures 3E-F).

\section{Activating Spinal NPY1R Alleviates Alloknesis in Aged Mice}

The NPY system's role in regulating mechanical itch was further assessed by intrathecally administering NPY or the 


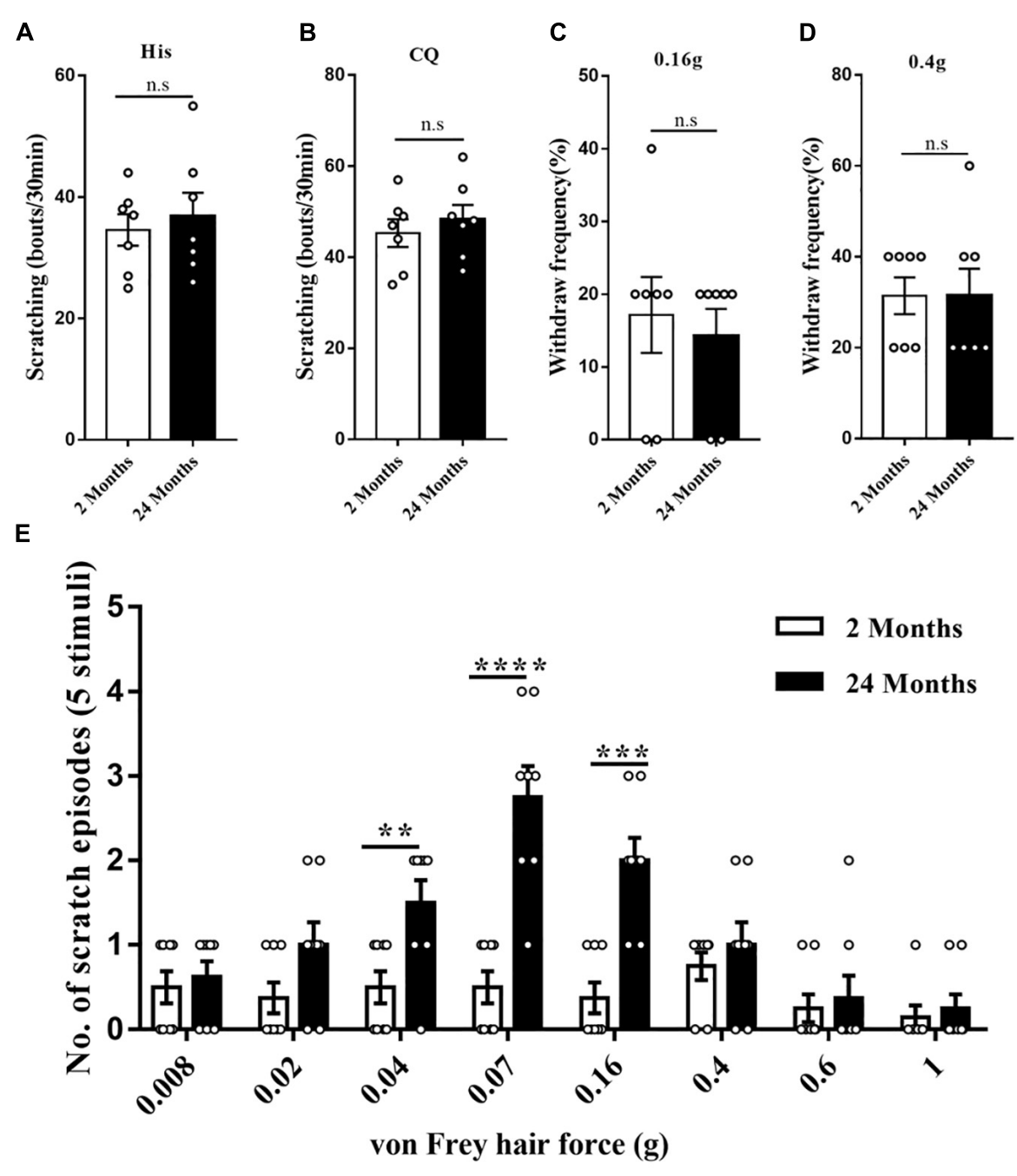

FIGURE 2 | The enhanced mechanical itch in aged mice. (A) Scratching responses to intradermal injections of histamine in young (2 months, $n=7,4$ male and 3 female) and aged (24 months, $n=7,4$ male and 3 female) mice. n.s, not significant, Student's $t$-test. (B) Scratching responses to intradermal injections of chloroquine in young ( 2 months, $n=7,4$ male and 3 female) and aged ( 24 months, $n=7,4$ male and 3 female) mice. n.s, not significant, Student's $t$-test. (C,D) Percent paw withdrawal responses of young and aged mice to 0.16 (C) and $0.4 \mathrm{~g}$ (D) von Frey filament stimulation. $n=7$ mice (4 male and 3 female) for each group. n.s, not significant, Student's $t$-test. (E) Alloknesis scores in young ( 2 months, $n=8,4$ male and 4 female) and aged ( 24 months, $n=8,4$ male and 4 female) mice. ${ }^{\star \star} P<0.01,{ }^{\star \star \star} P<0.001,{ }^{\star \star \star \star} P<0.0001,2$ versus 24 months, Student's $t$-test.

selective NPY1R agonist LP-NPY to the aged mice. The behavior assay showed that a supplement of NPY alleviated the mechanical itch in aged mice, especially in the 0.04 $(P=0.016), 0.07(P=0.00027)$, and $0.16 \mathrm{~g}(P=0.021)$ von Frey filaments. Moreover, activating NPY1R by LP-NPY reduced the scratching response to light mechanical stimuli (0.04 g: $P=0.040,0.07 \mathrm{~g}: P=0.000014$, and $0.16 \mathrm{~g}: P=0.0048)$ (Figure 4A). However, neither the injection of NPY nor LPNPY altered the reaction to classical pruritogens, namely, histamine and chloroquine, suggesting the unaffected chemical itch (His: 24 months + PBS vs. 24 months + NPY, $P=0.49$, 24 months + PBS vs. 24 months + LP-NPY, $P=0.88$; CQ: 24 months + PBS vs. 24 months + NPY, $P=0.30$, 24 months + PBS vs. 24 months + LP-NPY, $P=0.51$, Figures 4B,C).

\section{DISCUSSION}

In this study, we first demonstrated that aging down-regulated NPY in the spinal dorsal horn, that NPY deficiency evoked mechanical itch in young mice and intensified alloknesis in aged mice, and that pharmacological activation of the NPY/NPY1R signaling alleviated alloknesis in aged mice.

NPY was first abstracted from the pig brain in 1982 (Tatemoto et al., 1982). Accumulating studies had suggested that the NPY system, especially in the brain, was linked to the aging process and lifespan determination (Hua et al., 1991; Peng et al., 1993; Michalkiewicz et al., 2003; Gehrig et al., 2012). In general, NPY was down-regulated by aging in multiple brain regions, such as the cortex, hypothalamus, and striatum. In this study, the protein level of NPY was significantly decreased in the spinal dorsal horn 

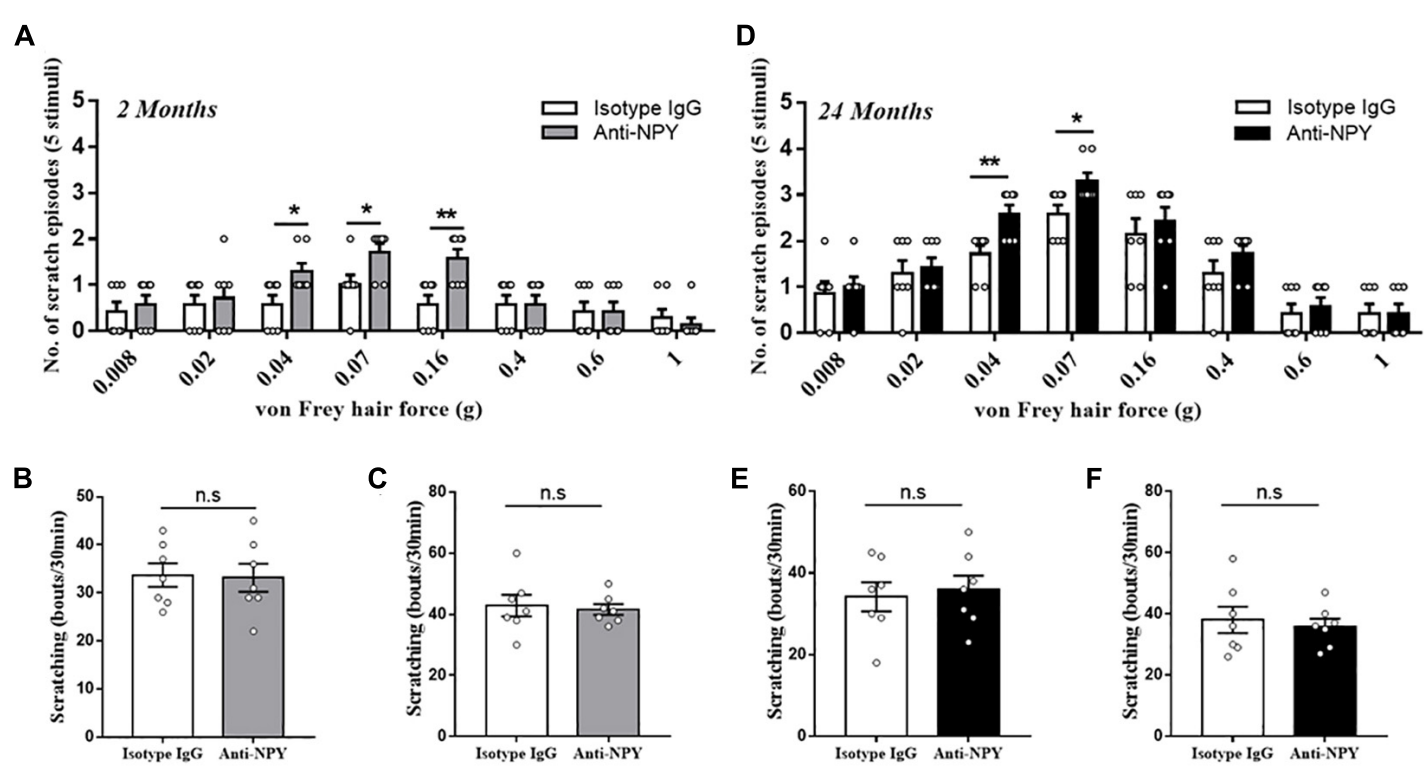

FIGURE 3 | Neutralizing NPY induced mechanical itch. (A) Alloknesis scores in young mice receiving an intrathecal injection of isotype lgG $(n=7,4$ male and 3 female) and anti-NPY IgG ( $n=7,4$ male and 3 female) mice. ${ }^{\star} P<0.05$, ${ }^{\star \star} P<0.01$, isotype lgG versus anti-NPY IgG, Student's $t$-test. (B) Scratching responses to intradermal injections of histamine in young mice receiving an intrathecal injection of isotype $\operatorname{lgG}(n=7,4$ male and 3 female) and anti-NPY IgG ( $n=7,4$ male and 3 female) mice. n.s, not significant, Student's t-test. (C) Scratching responses to intradermal injections of chloroquine in young mice receiving an intrathecal injection of isotype IgG ( $n=7,4$ male and 3 female) and anti-NPY IgG ( $n=7,4$ male and 3 female) mice. n.s, not significant, Student's $t$-test. (D) Alloknesis scores in aged mice receiving an intrathecal injection of isotype $\operatorname{lgG}\left(n=7,4\right.$ male and 3 female) and anti-NPY $\operatorname{lgG}\left(n=7,4\right.$ male and 3 female) mice. ${ }^{\star} P<0.05$, ${ }^{\star \star} P<0.01$, isotype $\operatorname{lgG}$ versus anti-NPY IgG, Student's t-test. (E) Scratching responses to intradermal injections of histamine in aged mice receiving an intrathecal injection of isotype lgG ( $n=7,4$ male and 3 female) and anti-NPY IgG ( $n=7,4$ male and 3 female) mice. n.s, not significant, Student's $t$-test. (F) Scratching responses to intradermal injections of chloroquine in aged mice receiving an intrathecal injection of isotype $\operatorname{lgG}(n=7,4$ male and 3 female) and anti-NPY IgG ( $n=7,4$ male and 3 female) mice. n.s, not significant, Student's $t$-test.

of aged mice. Moreover, these NPY-positive neurons presented the apoptotic character. Collectively, the deficiency of NPY might be the special label of aging in the CNS.

Actually, NPY defines a unique population of spinal inhibitory interneurons, most of which displayed a tonic firing pattern following current injection and expressed the markers of inhibitory interneuron. Moreover, $\mathrm{NPY}^{+}$interneurons gated mechanical itch specifically without affecting chemical itch (Bourane et al., 2015). In the present study, aged mice presented mechanical alloknesis together with degenerated $\mathrm{NPY}^{+}$neurons in the spinal dorsal horn, while the acute itch induced by classical pruritogens was not affected. This result suggested the correlation between NPY deficiency and alloknesis in aged mice. In aged mice, intrathecal injection of NPY alleviated mechanical itch suggesting that leaky gate control for mechanical itch within the spinal dorsal horn could be repaired by the supplement of NPY, while neutralizing spinal NPY by antibody intensified alloknesis. Previous studies also revealed the excitatory circuit for mechanical itch: $U \mathrm{cn} 3^{+}$interneurons received inputs from TLR5 ${ }^{+} \mathrm{A} \beta$ LTMRs and were directly gated by spinal $\mathrm{NPY}^{+}$interneurons (Pan et al., 2019). The changes within the excitatory circuit induced by aging and relative effects on aging alloknesis should be further investigated in the future. Considering that multiple neuropeptides serve as the gate for nociceptive information in the spinal cord (Kardon et al., 2014; Sun et al., 2017) and neuropeptides intend to degenerate during the aging process, the role of neuropeptides in somatosensory dysfunction among the aged population could be further investigated in the future.

The NPY receptors belong to G protein-coupled receptors (GPCR), including NPY1R, NPY2R, NPY4R, and NPY5R. Based on previous studies, this function of NPY might act by suppressing the excitability of excitatory intermediate neurons by the inhibitory signal from NPY1R (Acton et al., 2019). To determine the effective receptor for the NPY injection, the selective agonist for NPY1R was used to activate NPY1R specifically. The alleviation of alloknesis after the intrathecal application of LP-NPY in aged mice suggested, at least partly, that the responsive receptor for NPY was NPY1R. However, the chemical itch induced by histamine and chloroquine was not affected in the aged mice receiving the injection of NPY and LP-NPY. NPY1R was known as the major alloknesis-related NPY receptor now, so pharmacological modulation only targeted NPY1R in the present study. However, the responsive receptor might be multiple, so the effects of other NPY receptors on aging alloknesis should be studied further.

The somatosensory dysfunction, especially itch and pain, among the aged population burdens public health (Berger et al., 2013; Blyth and Noguchi, 2017; Qiu et al., 2020). Our findings illuminated that spinal NPY deficiency induced the loss of mechanical itch gating in aged mice, which shed new light on therapeutic strategies for the treatment of senile pruritus. 

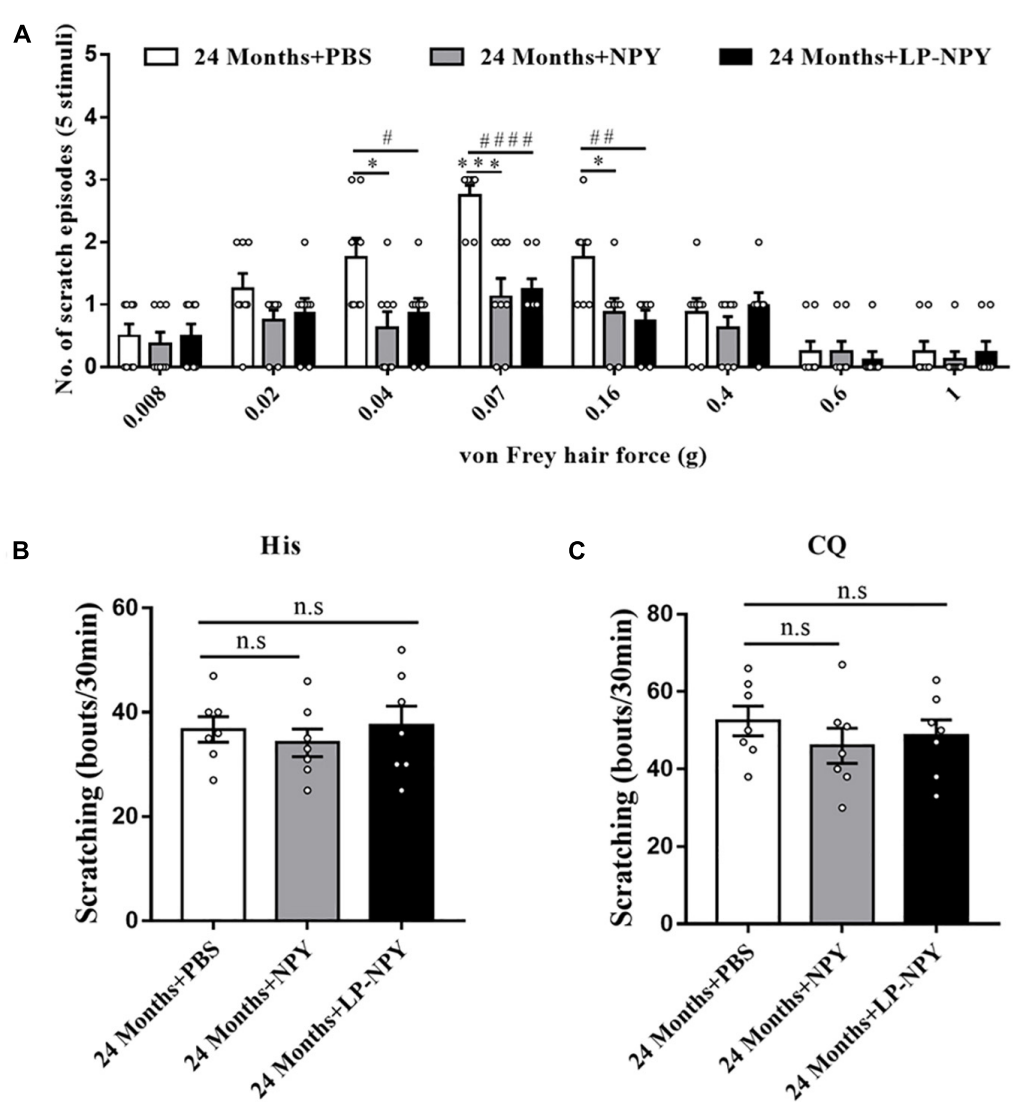

FIGURE 4 | Activation of spinal NPY1R alleviated the mechanical itch in aged mice. (A) Alloknesis scores in 24 months + PBS $(n=8), 24$ months + NPY $(n=8,4$ male and 4 female), and 24 months + LP-NPY ( $n=8,4$ male and 4 female) mice. ${ }^{*} P<0.05$, ${ }^{* * *} P<0.001,24$ months + PBS versus 24 months + NPY, Student's $t$

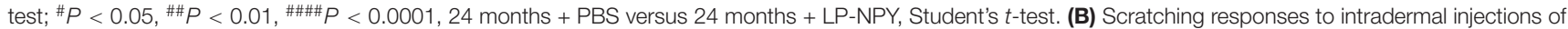
histamine in 24 months + PBS ( $n=7,4$ male and 3 female), 24 months + NPY ( $n=7,4$ male and 3 female), and 24 months + LP-NPY ( $n=7,4$ male and 3 female) mice. n.s, not significant, Student's t-test. (C) Scratching responses to intradermal injections of chloroquine in 24 months + PBS ( $n=7$, 4 male and 3 female), 24 months + NPY ( $n=7,4$ male and 3 female), and 24 months + LP-NPY ( $n=7,4$ male and 3 female) mice. n.s, not significant, Student's $t$-test.

\section{DATA AVAILABILITY STATEMENT}

The original contributions presented in the study are included in the article/supplementary material, further inquiries can be directed to the corresponding authors.

\section{ETHICS STATEMENT}

The animal study was reviewed and approved by Institutional Animal Care and Use Committee in Chinese Academy of Medical Sciences and Institute of Basic Medical Sciences.

\section{AUTHOR CONTRIBUTIONS}

HC and WS drafted the manuscript and performed the behavioral assessment of pain and itch. HC performed the immunofluorescence staining and western blotting. YC, LM, GX, WM, HZ, and JY performed the data analysis and behavioral assay. $\mathrm{CM}, \mathrm{XZ}$, and $\mathrm{YH}$ conceived the study, participated in its design, and helped to draft the manuscript. All authors read and approved the final manuscript.

\section{FUNDING}

This work was supported by the National Natural Science Foundation of China, grant numbers: \#81671098 (YH), \#81771205 (CM), and \#91632113 (CM), the Natural Science Foundation and Major Basic Research Program of Shanghai, grant numbers: \#16JC1420500 (CM) and \#16JC1420502 (CM), and the CAMS Innovation Fund for Medical Sciences (CIFMS), grant number: \#2017-I2M-3-008 (CM).

\section{ACKNOWLEDGMENTS}

We thank Dr. Wenyin Qiu, Xiaojin Qian, and Yongmei Chen in the Department of Anatomy, Histology and Embryology, Institute of Basic Medical Sciences and Chinese Academy of Medical Sciences for their technical assistance in immunohistochemistry. 


\section{REFERENCES}

Ward, J. R., and Bernhard, J. D. (2005). Willan's itch and other causes of pruritus in the elderly. Int J Dermatol 44, 267-273. doi: 10.1111/j.1365-4632.2004.02553.x

Valdes-Rodriguez, R., Stull, C., and Yosipovitch, G. (2015). Chronic pruritus in the elderly: pathophysiology, diagnosis and management. Drugs Aging 32, 201-215. doi: 10.1007/s40266-015-0246-0

Clerc, C. J., and Misery, L. (2017). A Literature Review of Senile Pruritus: From Diagnosis to Treatment. Acta Derm Venereol 97, 433-440. doi: 10.2340/ 00015555-2574

Chang, A. L., Wong, J. W., Endo, J. O., and Norman, R. A. (2013). Geriatric dermatology review: Major changes in skin function in older patients and their contribution to common clinical challenges. J Am Med Dir Assoc 14, 724-730. doi: 10.1016/j.jamda.2013.02.014

Bautista, D. M., Wilson, S. R., and Hoon, M. A. (2014). Why we scratch an itch: the molecules, cells and circuits of itch. Nat Neurosci 17, 175-182. doi: 10.1038/nn. 3619

Fukuoka, M., Miyachi, Y., and Ikoma, A. (2013). Mechanically evoked itch in humans. Pain 154, 897-904. doi: 10.1016/j.pain.2013.02.021

Koch, S. C., Acton, D., and Goulding, M. (2018). Spinal Circuits for Touch, Pain, and Itch. Annu Rev Physiol 80, 189-217. doi: 10.1146/annurev-physiol022516-034303

Yu, Y. Q., Barry, D. M., Hao, Y., Liu, X. T., and Chen, Z. F. (2017). Molecular and neural basis of contagious itch behavior in mice. Science 355, 1072-1076. doi: 10.1126/science.aak9748

Akiyama, T., Carstens, M. I., Ikoma, A., Cevikbas, F., Steinhoff, M., and Carstens, E. (2012). Mouse model of touch-evoked itch (alloknesis). J Invest Dermatol 132, 1886-1891. doi: 10.1038/jid.2012.52

Berger, T. G., Shive, M., and Harper, G. M. (2013). Pruritus in the older patient: a clinical review. Jama 310, 2443-2450. doi: 10.1001/jama.2013.282023

Feng, J., Luo, J., Yang, P., Du, J., Kim, B. S., and Hu, H. (2018). Piezo2 channelMerkel cell signaling modulates the conversion of touch to itch. Science 360, 530-533. doi: 10.1126/science.aar5703

Tatemoto, K., Carlquist, M., and Mutt, V. (1982). Neuropeptide Y-a novel brain peptide with structural similarities to peptide YY and pancreatic polypeptide. Nature 296, 659-660. doi: 10.1038/296659a0

Silva, A. P., Xapelli, S., Grouzmann, E., and Cavadas, C. (2005). The putative neuroprotective role of neuropeptide $\mathrm{Y}$ in the central nervous system. Curr Drug Targets CNS Neurol Disord 4, 331-347. doi: 10.2174/1568007054546153

Hsieh, Y. S., Chen, P. N., Yu, C. H., Liao, J. M., and Kuo, D. Y. (2013). The neuropeptide Y Y1 receptor knockdown modulates activator protein 1-involved feeding behavior in amphetamine-treated rats. Mol Brain 6, 46. doi: 10.1186/ 1756-6606-6-46

Bourane, S., Duan, B., Koch, S. C., Dalet, A., Britz, O., Garcia-Campmany, L., et al. (2015). Gate control of mechanical itch by a subpopulation of spinal cord interneurons. Science 350, 550-554. doi: 10.1126/science.aac8653

Pan, H., Fatima, M., Li, A., Lee, H., Cai, W., Horwitz, L., et al. (2019). Identification of a Spinal Circuit for Mechanical and Persistent Spontaneous Itch. Neuron 103, 1135.e-1149.e. 1135-1149.e1136,

Acton, D., Ren, X., Di Costanzo, S., Dalet, A., Bourane, S., Bertocchi, I., et al. (2019). Spinal Neuropeptide Y1 Receptor-Expressing Neurons Form an Essential
Excitatory Pathway for Mechanical Itch. Cell reports 28, 625.e-639.e. 625639.e626,

Fuhlendorff, J., Gether, U., Aakerlund, L., Langeland-Johansen, N., Thøgersen, H., Melberg, S. G., et al. (1990). [Leu31, Pro34]neuropeptide Y: a specific Y1 receptor agonist. Proceedings of the National Academy of Sciences of the United States of America 87, 182-186. doi: 10.1073/pnas.87.1.182

Hua, X. Y., Boublik, J. H., Spicer, M. A., Rivier, J. E., Brown, M. R., and Yaksh, T. L. (1991). The antinociceptive effects of spinally administered neuropeptide $Y$ in the rat: systematic studies on structure-activity relationship. J Pharmacol Exp Ther 258, 243-248.

Gavrieli, Y., Sherman, Y., and Ben-Sasson, S. A. (1992). Identification of programmed cell death in situ via specific labeling of nuclear DNA fragmentation. J Cell Biol 119, 493-501. doi: 10.1083/jcb.119.3.493

Gehrig, S. M., van der Poel, C., Sayer, T. A., Schertzer, J. D., Henstridge, D. C. Church, J. E., et al. (2012). Hsp72 preserves muscle function and slows progression of severe muscular dystrophy. Nature 484, 394-398. doi: 10.1038/ nature 10980

Peng, C., Chang, J. P., Yu, K. L., Wong, A. O., Van Goor, F., Peter, R. E., et al. (1993) Neuropeptide-Y stimulates growth hormone and gonadotropin-II secretion in the goldfish pituitary: involvement of both presynaptic and pituitary cell actions. Endocrinology 132, 1820-1829. doi: 10.1210/endo.132.4.846 2479

Michalkiewicz, M., Knestaut, K. M., Bytchkova, E. Y., and Michalkiewicz, T. (2003). Hypotension and reduced catecholamines in neuropeptide Y transgenic rats. Hypertension 41, 1056-1062. doi: 10.1161/01.hyp.0000066623.643 $68.4 \mathrm{e}$

Sun, S., Xu, Q., Guo, C., Guan, Y., Liu, Q., and Dong, X. (2017). Leaky Gate Model: Intensity-Dependent Coding of Pain and Itch in the Spinal Cord. Neuron 93, 840.e-853.e. 840-853.e845,

Kardon, A. P., Polgár, E., Hachisuka, J., Snyder, L. M., Cameron, D., Savage, S., et al. (2014). Dynorphin acts as a neuromodulator to inhibit itch in the dorsal horn of the spinal cord. Neuron 82, 573-586. doi: 10.1016/j.neuron.2014.02.046

Qiu, Y., Li, H., Yang, Z., Liu, Q., Wang, K., Li, R., et al. (2020). The prevalence and economic burden of pain on middle-aged and elderly Chinese people: results from the China health and retirement longitudinal study. BMC Health Serv Res 20:600.

Blyth, F. M., and Noguchi, N. (2017). Chronic musculoskeletal pain and its impact on older people. Best Pract Res Clin Rheumatol 31, 160-168. doi: 10.1016/j.berh. 2017.10.004

Conflict of Interest: The authors declare that the research was conducted in the absence of any commercial or financial relationships that could be construed as a potential conflict of interest.

Copyright (C) 2021 Cui, Su, Cao, Ma, Xu, Mou, Zhang, Yu, Ma, Zhang and Huang. This is an open-access article distributed under the terms of the Creative Commons Attribution License (CC BY). The use, distribution or reproduction in other forums is permitted, provided the original author(s) and the copyright owner(s) are credited and that the original publication in this journal is cited, in accordance with accepted academic practice. No use, distribution or reproduction is permitted which does not comply with these terms. 